\title{
Lower airway microbiota in children with Down syndrome compared to controls.
}

\author{
Mariska De Lausnay ${ }^{1}$, Stijn Verhulst ${ }^{2}$, Lieve Boel ${ }^{1}$, and Kim Van Hoorenbeeck ${ }^{3}$ \\ ${ }^{1}$ University Hospital Antwerp \\ ${ }^{2}$ Universitair Ziekenhuis Antwerpen \\ ${ }^{3}$ University of Antwerp
}

June 25, 2020

\begin{abstract}
Introduction: Children with Down syndrome (DS) often present with chronic or recurrent respiratory symptoms. This can be caused by anatomical and/or immunological predisposition. With this study, we aim to evaluate the differences in microbiota in the lower airways of patients with DS versus controls. Methods: All endoscopic procedures under general anesthesia in patients with DS were reviewed retrospectively. We compared the microbiological data from BAL samples (when available) to a cohort of children with chronic respiratory symptoms but without any other relevant medical history. Results: Endoscopic data were available for 65 DS patients, microbiological data for 47 out of 65 patients $(72 \%)$. The control group consisted of 150 children without significant underlying disease, who were matched for age and sex. Microbiological data were available for 135 out of 150 patients (90\%). Microorganisms were categorized and compared in both groups, with no statistical differences. Mostly present were typical bacteria such as H. influenzae, Moraxella catarrhalis, Streptococci and Staphylococci. Conclusion: There were no significant differences in presence of pathogens in the lower airways of children with DS and chronic respiratory symptoms, compared to controls with similar symptomatology.
\end{abstract}

\section{Introduction}

Trisomy 21 or Down syndrome (DS) is a relatively common human genetic disorder. It is well known for the characteristic dysmorphic features, cognitive impairment and hypotonia, but patients also have a high incidence of congenital anomalies in several organ systems: most frequently affected are the heart, gastrointestinal tract and respiratory system. These features makes them more prone to airway obstruction and other respiratory problems . Children with DS are more vulnerable for infections, probably multifactorial in origin but an altered immune status has also been described. Studies show that respiratory problems such as upper and lower respiratory tract infections (e.g. otitis media, tonsillitis, pneumonia, bronchiolitis) are the most common admission diagnoses in this patient population.

In a previous study, we focused on the anatomy of the lower airways and reviewed endoscopic results to compare the prevalence of airway anomalies in a population of children with DS with a control group (both with chronic or recurrent respiratory symptoms). In conclusion, we found a significantly higher prevalence of both isolated and combined airway malformations compared to controls (with the occurrence of one or more anomalies in $71 \%$ of endoscopies in DS versus $32 \%$ of controls, $\mathrm{p}<0.001$ ). This confirmed the conclusions of previous small-scale studies. However, little is known about the microbiota of these children.

In the past, the lungs were thought to be a sterile environment. We now know that the lower airways are a complex ecosystem, with a rich mucosal flora that differs from the microbiome in the upper airways (which in 
turn shows major differences in microbiome when comparing for example the nasal and oral cavity). However, examining the lower airway microbiota is challenging. This is because of the technical difficulties in accurate sampling and also the extremely low bacterial burden in healthy lungs. While the upper respiratory tract has a high bacterial burden due to continuous exposure via ingestion and inhalation, this is 100 to 10.000 times lower in the lower airways. Although the vocal cords act as a barrier, there are still continuous bacterial challenges due to microaspiration, postnasal drip, regurgitation, hematogenous spread and inhalation. In addition to these exposures, use of antibiotics or steroids, coinfection with viruses and availability of nutrients can play important roles in the shaping of a specific microbiome and immunological fenotypes .

Studies in children with chronic cough and diagnosis of protracted bacterial bronchitis, have shown clinically significant levels of the following microorganisms in BAL (bronchoalveolar lavage) fluid: Haemophilus influenzae, Streptococcus pneumoniae, Moraxella catarrhalis and Staphylococcus aureus. These pathogens were associated with altered bacterial community structure (lower alpha diversity of the respiratory microbiota), higher bacterial biomass and higher inflammatory parameters such as neutrophil percentage and several interleukins, compared to controls .

A few case reports from the 1980-1990's exist that describe atypically severe lower airway infections in children with DS. Cant et al described a series of 4 DS patients presenting with acute bacterial tracheitis. Three of them had cultures positive for $\mathrm{H}$. influenzae and one remained sterile, probably due to sampling done after several doses of antibiotic therapy. All of them were severely ill and required mechanical ventilation. Orlicek et al described another series of 3 young DS patients with severe bilateral pneumonia with Mycoplasma pneumonia as causative agent. A report by Winters et al described a case of lethal pneumonia in a DS patient caused by Bordetella bronchiseptica, which is a microorganism generally found in animal species but in rare cases also causes severe infections in immunocompromised patients. These reports suggest a susceptibility in DS patients for atypical microorganisms or atypical course of infection. However, these reports are rare and non-recent. Further data are lacking.

Therefore, the aim of this study is to compare microbiological data (colonization) from the lower airways by endoscopic investigation in a cohort of children with DS from our institution, to a group of controls without significant medical history. This may deliver valuable information for future decision-making in terms of treatment.

\section{Methods}

We used databases from our previous study, where a retrospective chart review was performed of all endoscopic procedures (flexible bronchoscopy and flexible / rigid laryngoscopy, all under general anesthesia with spontaneous breathing) in pediatric patients with DS from April 2011 until June 2019. As a control group, we selected children without significant underlying disorder undergoing endoscopy for similar indications (selected from charts dating from January 2012 until January 2015).

In both groups, we checked for each patient whether a BAL sample for microbiological investigation was obtained during the endoscopic procedure. Of course this is only possible if a bronchoscopy was performed. If this was the case, results of cultures and / or PCR testing were listed. All positive cultures (even with small numbers of colony forming units per $\mathrm{ml}$ ) were taken into account, in order to determine colonization. These results were subsequently categorized and compared by statistical analysis in SPSS. We used a Chi square test to compare the different categories of microbiological results and detect if the differences between both cohorts are statistically significant. The level of statistical significance was set at P [?] 0.05 . 


\section{Results}

At present, over 300 children with DS are followed-up in our center by a multidisciplinary team. For 65 of them data on airway endoscopy are available. On average, a broncho- or laryngoscopy was performed at the age of 2.89 years, with a range from 2 months to 17 years. Most of the patients were male (63\%). Our control group consists of 150 children with respiratory symptoms that warranted endoscopic evaluation, but without additional underlying conditions. They were matched in terms of age and sex with our DS group: the median age of endoscopy was 3.5 years $(\mathrm{p}=0.418), 63 \%$ were boys $(\mathrm{p}=0.971)$.

Samples for cultures and PCR testing were obtained in 47 of the DS patients $(47 / 65=72 \%)$ and in 135 of our control patients $(135 / 150=90 \%)$. In most of these patients, cultures showed multiple organisms (for example in $64 \%$ of the DS group). The encountered microorganisms were labeled as typical bacteria, atypical bacteria or other (mostly viruses) [table 1], based on experience and previous studies . We then attributed each patient to a category, depending on the microbiological data [table 2]: 1) sterile or only presence of commensals, 2) only typical bacteria present, 3) only atypical bacteria present, and 4) a combination of both typical and atypical bacteria. When comparing the DS to the control group, we found similar microorganisms. Also the percentages of encountered categories were comparable, no statistically significant differences were found [table 2].

\section{Discussion}

Even though children with DS are more prone to infections (often originating in the respiratory tract) due to several predisposing factors, this inquiry concludes that the pathogens encountered in bronchoalveolar lavage fluids are similar compared to controls. Most frequently, cultures showed growth of typical bacteria such as Haemophilus influenza, catarrhalis species and Streptococcus pneumoniae in both groups. This was also the case in several studies of children with protracted bacterial bronchitis . The previously mentioned case reports about atypical pathogens in DS therefore seem to be rather exceptional. When treating patients with DS and (chronic) lower airway infections, antibiotic therapy should not necessarily be adjusted to a more broad spectrum than in children without underlying conditions. However, the multiple comorbidities in these patients and at times atypical or more severe course of infections vindicate a thorough evaluation and adequate treatment.

\section{Conclusion}

As expected, no significant differences were found concerning the lower airway microbiota of children with DS compared to controls. Based on these (albeit limited) data, we conclude that the higher infectious burden in children with DS may be explained by the immunological and anatomical abnormalities. The type of pathogens however are similar.

\section{References:}

1. Stoll C, Dott B, Alembik Y, Roth MP. Associated congenital anomalies among cases with Down syndrome. Eur J Med Genet : sn, 2015 Dec;58(12):674-80.

2. Watts R, Vyas H. An overview of respiratory problems in children with Down's syndrome. 2013 Oct, Arch Dis Child., pp. 98(10):812-7.

3. Bloemers BL, Broers CJ, Bont L, Weijerman ME, Gemke RJ, van Furth AM. Increased risk of respiratory tract infections in children with Down syndrome: the consequence of an altered immune system. Microbes Infect. 2010 Oct;12(11):799-808. 
4. Ram G, Chinen J. Infections and immunodeficiency in Down syndrome. Clin Exp Immunol. 2011 Apr;164(1):9-16.

5. Alsubie HS, Rosen D. The evaluation and management of respiratory disease in children with Down syndrome (DS). Paediatr Respir Rev. 2017;26:49-54.

6. Fitzgerald P, Leonard H, Pikora TJ, Bourke J, Hammond G.Hospital admissions in children with down syndrome: experience of a population-based cohort followed from birth. PLoS One. 2013 Aug 13;8(8):e70401

7. So SA, Urbano RC, Hodapp RM. Hospitalizations of infants and young children with Down syndrome: evidence from inpatient person-records from a statewide administrative database. J Intellect Disabil Res. 2007 Dec;51(Pt 12):1030-8.

8. De Lausnay M, Verhulst S, Boel L, Wojciechowski M, Boudewyns A, Van Hoorenbeeck K. The prevalence of lower airway anomalies in children with Down syndrome compared to controls. Pediatr Pulmonol. 2020 May;55(5):1259-1263.

9. Bertrand P, Navarro H, Caussade S, Holmgren N, Sánchez I.Airway anomalies in children with Down syndrome: endoscopic findings. Pediatr Pulmonol. 2003;36(2):137-141.

10. Hamilton J, Yaneza MM, Clement WA, Kubba H. The prevalence of airway problems in children with Down's syndrome. Int J Pediatr Otorhinolaryngol. 2016;81:1-4.

11. J, Pravit. Bronchoscopic findings in Down syndrome children with respiratory problems. J Med Assoc Thai. 2014;97:S159-S163.

12. Wu BG, Segal LN. Lung Microbiota and Its Impact on the Mucosal Immune Phenotype. Microbiol Spectr. 2017 Jun;5(3).

13. Marsh RL, Smith-Vaughan HC, Chen ACH, Marchant JM, Yerkovich ST, Gibson PG, Pizzutto SJ, Hodge S, Upham JW, Chang AB. Multiple Respiratory Microbiota Profiles Are Associated With Lower Airway Inflammation in Children With Protracted Bacterial Bronchitis.Chest. 2019 Jan 17. pii: S0012-3692(19)30010-8.

14. Ahmed B, Cox MJ, Cuthbertson L, James PL, Cookson WOC, Davies JC, Moffatt MF, Bush A. Comparison of the upper and lower airway microbiota in children with chronic lung diseases. PLoS One. 2018 Aug 2;13(8):e0201156.

15. Verhulst S, Boel L, Van Hoorenbeeck K. Protracted bacterial bronchitis: bronchial aspirate versus bronchoalveolar lavage findings: a single-centre retrospective study. BMJ Paediatr Open. 2019 Jun 20;3(1):e000507.

16. Cant AJ, Gibson PJ, West RJ. Bacterial tracheitis in Down's syndrome. Arch Dis Child. 1987 Sep;62(9):962-3.

17. Orlicek SL, Walker MS, Kuhls TL. Severe mycoplasma pneumonia in young children with Down syndrome. Clin Pediatr (Phila). 1992 Jul;31(7):409-12.

18. Winters JL, O'Connor WN, Broughton RA, Noonan JA.Bordetella bronchiseptica pneumonia in a patient with Down syndrome: a case report and review. Pediatrics. 1992 Jun;89(6 Pt 2):1262-5.

\section{Hosted file}

Microbiotia DS Table 1.docx available at https://authorea.com/users/336844/articles/462517lower-airway-microbiota-in-children-with-down-syndrome-compared-to-controls

Hosted file 
Microbiotia DS Table 2.docx available at https://authorea.com/users/336844/articles/462517lower-airway-microbiota-in-children-with-down-syndrome-compared-to-controls 